\title{
Conditions for growth and oil production of marine microalga Nannochloropsis sp.
}

\author{
Nyuk-Min Chong, Shih-TsungYu, Chang-Yu Lo
}

Abstract-The purpose of this study was to explore the ways that could enhance the productivity of oil by a marine microalga Nannochloropsis sp. Microalgae have the characteristics of rapid growth with ample accumulation of oil in their cells. Algae are photoautotrophs that grow on carbon dioxide. With the yield of oil rich biomass, cultivation of microalgae suits the noble purposes of pollutant reduction and renewable energy production. In this study, Nannochloropsis sp., which is known for its large biomass yield with high cell oil content, was cultured in batch reactors in the Walne medium. Light intensity was controlled at 11000 Lux and culture temperature was set at $32 \pm$ $2^{\circ} \mathrm{C}$. Sodium bicarbonate was the carbon source and sodium nitrate was the nitrogen source, both of which were variables for the determination of maximal oil productivity. pH was held at controlled and un-controlled states. Test results showed that with sodium bicarbonate and sodium nitrate concentration of $12 \mathrm{~g}$ $\mathrm{HCO}_{3}{ }^{-} \mathrm{L}^{-1}$ and $0.5 \mathrm{~g} \mathrm{NO}_{3}-\mathrm{N} \mathrm{L}^{-1}$, respectively, the highest quantity of biomass was obtained after seven days of culture. However, the

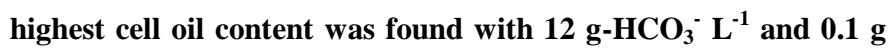
$\mathrm{NO}_{3}-\mathrm{N} \mathrm{L}^{-1}$ of bicarbonate and nitrate, respectively. The outputs of cell mass concentration and oil content were 0.8 g-dry-wt $L^{-1}$

\footnotetext{
Nyuk-Min, Chong, Shih-Tsung, Yu and Chang-Yu, Lo

Department of Environmental Engineering, DaYeh University

Taiwan.
}

and $17 \%$ for the high nitrate culture, while cell mass and oil content for the low nitrate case were 0.5 g-dry-wt $L^{-1}$ and $38 \%$. Comparing oil production with the high nitrate feed, the low nitrate feed had an advantage in oil production by approximately 0.05 g-oil $\mathbf{L}^{-1}(+40 \%)$. Higher nitrogen supply is diverted to synthesizing proteinaceous cell mass instead of lipid. Increase in pH approaching 9.5 when bicarbonate was consumed did not hinder Nannochloropsis growth; control of medium $\mathrm{pH}$ below the ceiling of 9.0 resulted in lower performances in both biomass production and oil content therein. Bicarbonate and nitrate were found to be the controlling factors of oil production from the cultivation of microalga Nannochloropsis sp.

Keywords-biomass oil, renewable energy, microalgae, carbondioxide mitigation, optimization

\section{Acknowledgment}

The work reported in this presentation was supported in parts by the research grant NSC 100-2632-B-212-001-MY3 provided by the Ministry of Science and Technology of Taiwan, the Republic of China.

\section{References}

[1] M. Takagi and T. Yosida, "Effect of salt concentration on interacellular acclumulation of lipids and tgriacylglyceride in morine microalgae Dunaliella cells," J Biosci. Bioengr, vol. 101, pp. 223-226, 2006.

[2] E. W. Becker, Microalgae: Biotechnology and microbiology. Cambridge University Press, 1994.

[3] D. H. Turpin, "Effect of inorganic $\mathrm{N}$ availability on algal photosynthesis and carbon metabolism," J. Phycology, vol. 27, pp. 14-20, 1991. 
Proc. of the Fourth International Conference on Advances in Civil, Structural and Environmental Engineering - ACSEE 2016. Copyright $(\odot$ Institute of Research Engineers and Doctors. All rights reserved. ISBN: 978-1-63248-114-6 doi: 10.15224/ 978-1-63248-114-6-26

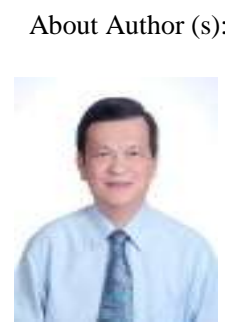

Prof. Chong $(\mathrm{PhD})$ has a professional career in environmental engineering for more than 30 years, including field engineering and professorship in universities. Prof. Chong's main area of research is environmental biotechnology, and also has teamed with Prof. Yu in the research of biomass energy production.

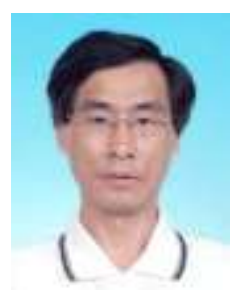

Prof. $\mathrm{Yu}(\mathrm{PhD})$ has been associated with the energy industry fir 10 years and for 20 years now is working as a professor in university. Prof. Yu's research interest is in the area of renewable energy mainly biomass energy. Mr. CY Lo is Prof. Yu's graduate student, now pursuing careers in environmental engineering. 